
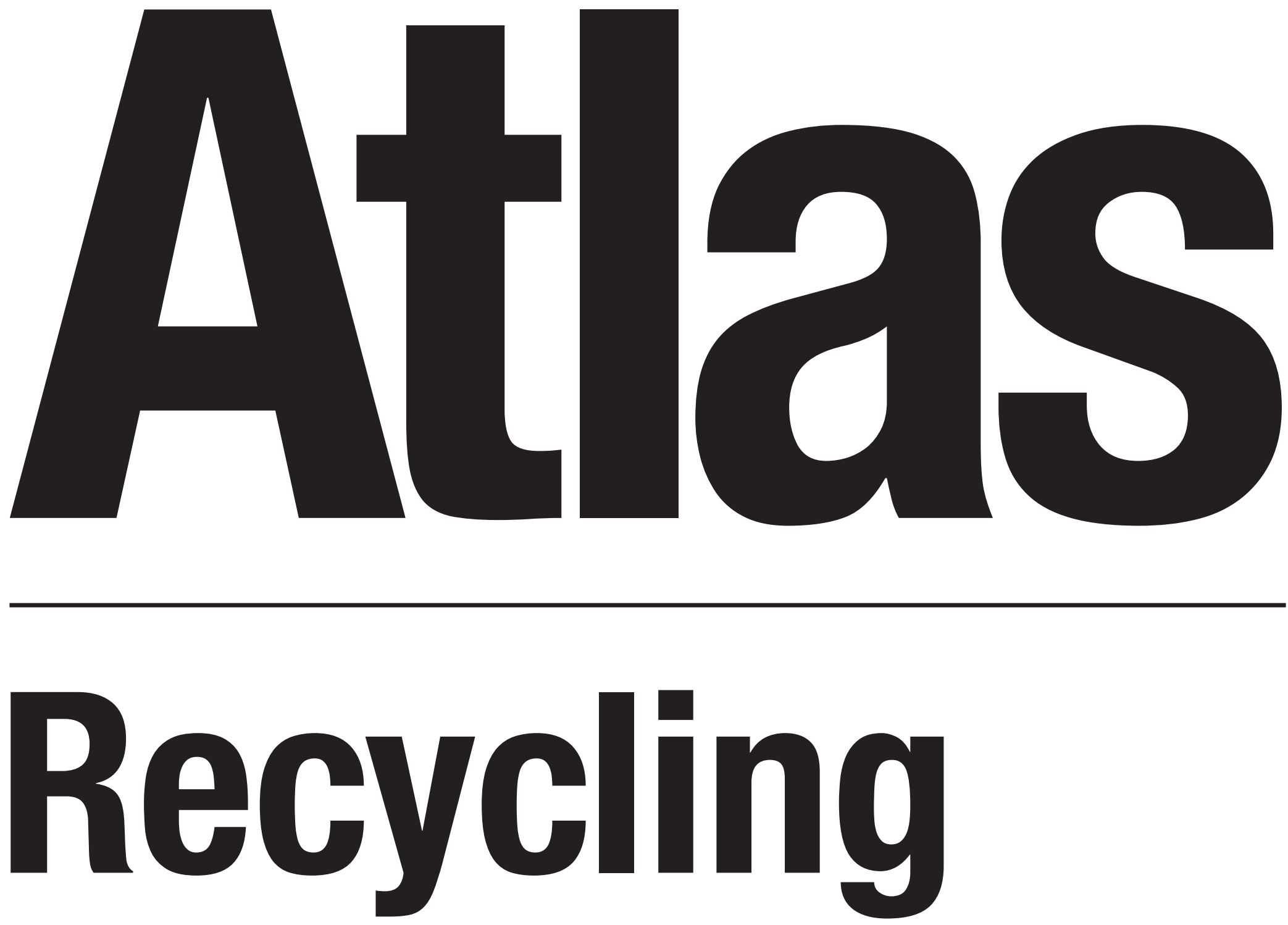

Gebäude als Materialressource

\title{
Annette Hillebrandt
}

Petra Riegler-Floors

Anja Rosen

Johanna-Katharina Seggewies

Edition DETAIL 


\section{Autorinnen und Autoren}

Annette Hillebrandt

Univ.-Prof. Dipl.-Ing. Architektin

Petra Riegler-Floors

Prof. Dipl.-Ing. Architektin

Anja Rosen

Dr.-Ing. M. A. Architektin

Johanna-Katharina Seggewies

M.Sc. M. A. Architektin

wissenschaftliche Hilfskraft:

Julia Blasius, M. Sc.

alle:

Bergische Universität Wuppertal,

Fakultät für Architektur und Bauingenieurwesen,

Lehrstuhl Baukonstruktion I Entwurf I Materialkunde

Forschungsschwerpunkt:

Kreislaufpotenziale von Konstruktionen und

Materialien im Hochbau mit Fachbeiträgen von:

Prof. Dr. Günther Bachmann

Rat für Nachhaltige Entwicklung, Berlin

Prof. Dipl.-Ing. Markus Binder

Hochschule für Technik Stuttgart

Fachgebiet Integrierte Gebäudetechnik

CAPE climate architecture physics energy,

Esslingen/Schwäbisch Hall

Univ.-Prof. Dr.-Ing. Manfred Helmus

Bergische Universität Wuppertal,

Lehr- und Forschungsgebiet Baubetrieb und Bauwirtschaft

Univ.-Prof. Holger Hoffmann

Bergische Universität Wuppertal,

Lehrstuhl Darstellungsmethodik und Entwerfen

Dipl.-Ing. Mag. Thomas Kasper

Porr Umwelttechnik GmbH, Wien

Dipl.-Ing. Holger Kesting

Bergische Universität Wuppertal,

Lehr- und Forschungsgebiet Baubetrieb und Bauwirtschaft

Dipl.-Ing. Architekt Thomas Matthias Romm

forschen planen bauen, Wien

Dipl.-Ing. Michael Wengert,

B. Eng. Tobias Edelmann

Pfeil + Koch ingenieurgesellschaft, Stuttgart

ISBN: 978-3-95553-415-8 (Print)

ISBN: 978-3-95553-416-5 (E-Book)

Redaktion und Lektorat:

Steffi Lenzen (Projektleitung),

Jana Rackwitz, Daniel Reisch

Redaktionelle Mitarbeit:

Michaela Linder, Laura Oberhofer,

Charlotte Petereit, Heike Werner

Endlektorat:

Carola Jacob-Ritz, München (DE)

Zeichnungen:

Marion Griese, Ralph Donhauser

Coverdesign:

Wiegand von Hartmann GbR, München (DE)

Herstellung /DTP:

Simone Soesters

Repro:

ludwig:media, Zell am See

Druck und Bindung:

Grafisches Centrum Cuno GmbH \& Co. KG, Calbe (DE)

Papier:

Remake Favini (Umschlag), Profibulk (Innenteil)

๑ 2021, zweite, korrigierte Auflage

Detail Business Information $\mathrm{GmbH}$, München

detail.de
Dieses Werk ist urheberrechtlich geschützt. Die dadurch begründeten Rechte, insbesondere die der Übersetzung, des Nachdrucks, des Vortrags, der Entnahme von Abbildungen und Tabellen, der Funksendung, der Mikroverfilmung oder der Vervielfältigung auf anderen Wegen und der Speicherung in Datenverarbeitungsanlagen, bleiben, auch bei nur auszugsweiser Verwertung, vorbehalten. Eine Vervielfältigung dieses Werks ist auch im Einzelfall nur in den Grenzen der gesetzlichen Bestimmungen des Urheberrechtsgesetzes in der jeweils geltenden Fassung zulässig. Sie ist grundsätzlich vergütungspflichtig. Zuwiderhandlungen unterliegen den Strafbestimmungen des Urheberrechts.

Die Inhalte dieses Fachbuchs wurden nach bestem Wissen und Gewissen sowie mit größter Sorgfalt recherchiert und erarbeitet. Sie bilden in Teilen einen Forschungsstand ab und sind daher nicht alle in der Praxis erprobt. Rechtliche Ansprüche können aus dem Inhalt dieses Buchs nicht abgeleitet werden.

Bibliografische Information der Deutschen Nationalbibliothek Die Deutsche Nationalbibliothek verzeichnet diese Publikation in der Deutschen Nationalbibliografie; detaillierte bibliografische Daten sind im Internet abrufbar unter http://dnb-nb.de.

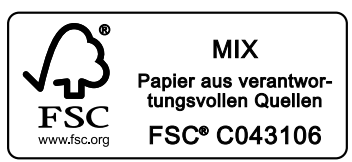

